\title{
A LIAPUNOV FUNCTIONAL FOR LINEAR VOLTERRA INTEGRODIFFERENTIAL EQUATIONS
}

\author{
BY
}

D. L. ABRAHAMSON (Brown University)

AND

E. F. INFANTE (National Science Foundation and Brown University)

\begin{abstract}
Liapunov functionals of quadratic form have been used extensively for the study of the stability properties of linear ordinary, functional and partial differential equations. In this paper, a quadratic functional $V$ is constructed for the linear Volterra integrodifferential equation

$$
\begin{gathered}
\dot{x}(t)=A x(t)+\int_{0}^{T} B(t-\tau) x(\tau) d \tau, \quad t \geq t_{0}, \\
x(t)=f(t), \quad 0 \leq t \leq t_{0} .
\end{gathered}
$$

This functional, and its derivative $\dot{V}$, is more general than previously constructed ones and still retains desirable computational qualities; moreover, it represents a natural generalization of the Liapunov function for ordinary differential equations. The method of construction used suggests functionals which are useful for more general equations.
\end{abstract}

1. Introduction. For ordinary differential equations of the form $\dot{x}(t)=A x(t), x(t) \in \mathbb{R}^{n}$, it is well known that stability, perturbation and asymptotic properties can be obtained through the use of Liapunov functionals of the form $V=x^{T} M x$. Indeed, if $A$ is asymptotically stable a positive definite symmetric matrix $M$ can be obtained as the solution of the Liapunov algebraic equation $A^{T} M+M A=-W$ for any positive definite matrix $W$; moreover, one has the familiar result that $M=\int_{0}^{\infty} e^{A^{T} s} W e^{A s} d s$ and that $\dot{V}=-x^{T} W x$. Since $V$ and $\dot{V}$ are quadratic forms, it is easy to obtain an estimate of the type $\dot{V}(t) \leq-2 \delta V(t)$ for some positive number $\delta$ and therefore to obtain a decay estimate via the Gronwall inequality. It is well known that this estimate can be made arbitrarily close to the best possible estimate; furthermore, $V^{1 / 2}$ is a norm equivalnet to the Euclidian norm in $\mathbb{R}^{n}$. The simplicity, computability and sharpness of these results are central to a number of useful techniques in asymptotic and perturbation analysis of ordinary differential equations.

In this paper we present a quadratic Liapunov functional for a linear Volterra integrodifferential equation. Because the function space involved is not $\mathbb{R}^{n}$, it is not clear what quadratic functionals should be chosen that will lead to sharp results yet remain easily

* Received March 10, 1982. The research of D. L. A. was partially supported by the Air Force Office of Scientific Research under contract AF-AFOSR 81-0198; that of E. F. I. was partially supported by the National Science Foundation under contract MCS-79-05774-02. 
computable. We follow the methodology suggested by the ordinary differential equation case and choose what seems an appropriate quadratic form $V$ for the functional. Computation of the rate of change of the functional along the solutions of the equation yields another functional $\dot{V}$; for the sake of computability and comparability, conditions are imposed on $\dot{V}$, which are then reflected in the form $V$. In the course of this analysis an auxiliary equation, the natural generalization of the Liapunov algebraic equation, arises and is analyzed. The end result is a theorem that states the existence of Liapunov functionals of the desired form which give sharp estimates of decay rates.

We consider the equation

$$
\dot{x}(t)=A(t) x(t)+\int_{0}^{t} K(t, \tau) x(\tau) d \tau, \quad t \geq t_{0} \geq 0, \quad x(t) \in \mathbb{R}^{n},
$$

with $x(t)=f(t)$ for $f \in C\left[0, t_{0}\right]$. Miller [1] and others have shown the desirability and usefulness of considering such equations with $t_{0}>0$. Denoting by $x\left(\cdot, t_{0}, f\right)$ the solution of this equation with initial data $f \in C\left[0, t_{0}\right]$, we say that the equation is stable if for every $t_{0} \geq 0$ and $\varepsilon>0$ there exists a $\delta=\delta\left(t_{0}, \varepsilon\right)>0$ such that $|f(t)|<\delta, 0 \leq t \leq t_{0}$, implies $\left|x\left(t, t_{0}, f\right)\right|<\varepsilon$ for $t \geq t_{0}$; the equation is said to be uniformly stable if it is stable and $\delta=\delta(\varepsilon)$ can be chosen independent of $t_{0} \geq 0$.

For the simpler equation

$$
\dot{x}(t)=A x(t)+\int_{0}^{t} B(t-\tau) x(\tau) d \tau, \quad t \geq t_{0} \geq 0, \quad x(t) \in \mathbb{R}^{n},
$$

where $A$ is a constant matrix and the convolution kernel $B$ is integrable, characterizations of stability properties have been obtained by Miller [1] and Grossman and Miller [2]. Seifert $[3,4]$ and Grimmer and Seifert $[5]$ have studied this equation through the use of the very simple quadratic Liapunov functional $x^{T} J x$, under the assumption that $A$ is asymptotically stable and that $A^{T} J+J A$ is negative definite. Burton $[6,7]$ has studied Eqs. (1.1) and (1.2) using more general functionals, generally not quadratic ones, functionals which include the history of the solution. The construction of quadratic Liapunov functionals for linear problems in Hilbert spaces has been pursued extensively by Infante and others $[8,9,10,11]$. This latter approach has motivated this investigation; indeed, the results here generalize those in [9]. In Sec. 2 we construct a quadractic Liapunov functional for Eq. (1.2); in Sec. 3 we exhibit a functional which is useful for the study of Eq. (1.1) and present an example of its use.

We wish to thank our colleague Professor John Mallet-Paret for some very useful suggestions which have been incorporated in this paper.

2. Equations with kernels of convolution type. Consider the equation

$$
\dot{x}(t)=A x(t)+\int_{0}^{t} B(t-\tau) x(\tau) d \tau, \quad t \geq t_{0} \geq 0, \quad x(t) \in \mathbb{R}^{n},
$$

where $A$ and $B$ are $n \times n$ matrices with $A$ constant and $B$ locally integrable. For each $t \geq t_{0}$ we consider the pair $\left(x_{t}(0), x_{t}\right) \in \mathbb{R}^{n} \times L_{2}[-t$, 0$]$, where $x_{t}(\theta)=x(t+\theta)$ for $-t \leq \theta \leq 0$. Associated with this equation is the equation

$$
\dot{y}(t)=(A+\delta I) y(t)+\int_{0}^{t} e^{\delta(t-\tau)} B(t-\tau) y(\tau) d \tau, \quad t \geq t_{0} \geq 0,
$$


where $\delta \in \mathbb{R}$, which is obtained from (2.1) through the change of variables $y(t)=e^{\delta t} x(t)$.

In the sequel, we shall use a representation of the solutions of (2.1) similar to those given by Miller [12] and Hale [13]. Let $S$ be the solution of the matrix initial value problem

$$
\dot{S}(t)=S(t) A+\int_{0}^{t} S(t-\tau) B(\tau) d \tau, \quad t \geq 0, \quad S(0)=I,
$$

and let $S(\theta)=0$ for $\theta<0$. Then we have

Lemma 2.1. Suppose $x(t)$ is a solution of (2.1) for $t \geq t_{0}$. Then if $t \geq t_{0}$ and $\rho \geq 0$,

$$
x_{t+\rho}(0)=S(\rho) x_{t}(0)+\int_{-t}^{0} \int_{-\theta}^{\infty} S(\rho-v-\theta) B(v) x_{t}(\theta) d v d \theta .
$$

The matrix function $S_{\delta}(t)=e^{\delta t} S(t)$ is, of course, related to Eq. (2.2).

The quadratic forms to be used as candidates for Liapunov functionals for Eq. (2.1) are of the type

$$
\begin{aligned}
V\left(t, x_{t}(0), x_{t}\right)=x_{t}^{T}(0) L x_{t}(0) & +\int_{-t}^{0} x_{t}^{T}(\theta) H(\theta) x_{t}(\theta) d \theta \\
& +2 x_{t}^{T}(0) \int_{-t}^{0} N(\theta) x_{t}(\theta) d \theta+\int_{-t}^{0} \int_{-t}^{0} x_{t}^{T}(\theta) P(\theta, \eta) x_{t}(\eta) d \eta d \theta,
\end{aligned}
$$

where $L$ and $H(\theta),-t \leq \theta \leq 0$, are symmetric positive definite matrices and $P(\theta, \eta)=$ $P^{T}(\eta, \theta)$ for $-t \leq \theta, \eta \leq 0$. This quadratic form is natural for our equation. The rate of change of $V$ along the solutions of (2.1) for $t \geq t_{0}$ can be obtained, after a lengthy but straightforward computation, as

$$
\begin{aligned}
\dot{V}(t, & \left.x_{t}(0), x_{t}\right)=x_{t}^{T}(0)\left[A^{T} L+L A+H(0)+N(0)+N^{T}(0)\right] x_{t}(0) \\
& -\int_{-t}^{0} x_{t}^{T}(\theta) H^{\prime}(\theta) x_{t}(\theta) d \theta+2 x_{t}^{T}(0) \int_{-t}^{0}\left[L B(-\eta)+A^{T} N(\eta)-N^{\prime}(\eta)+P(0, \eta) x_{t}(\eta) d \eta\right. \\
& +\int_{-t}^{0} \int_{-t}^{0} x_{t}^{T}(\theta)\left[B^{T}(-\theta) N(\eta)+N^{T}(\theta) B(-\eta)-\frac{\partial P(\theta, \eta)}{\partial \theta}-\frac{\partial P(\theta, \eta)}{\partial \eta}\right] x_{t}(\eta) d \eta d \theta
\end{aligned}
$$

This quadratic form is of the same type as (2.5). Unfortunately, at this level of generality it is difficult, if not impossible, to obtain simple computable inequalities between $V$ and $\dot{V}$.

In $[3,4,5,6,7]$ this difficulty has led one to choose all or several of the matrix functions $H, N$ and $P$ to be identically zero, yielding rather restrictive results. The analogy to the case of the simple ordinary differential equation $\dot{x}=A x$ with Liapunov functional $V=x^{T} J x$ is to pick a particularly simple form $J=J^{T}$, say diagonal, and to insist that the form $E=-\left(A^{T} J+J A\right)$ be positive definite. It is well known that, in general, the stability results thus obtained for the solutions of the differential equation will be most restrictive. Our viewpoint is the opposite one. We choose a particularly simple form for the matrix $E$ and thus obtain the matrix $J$. This is precisely the viewpoint successfully employed in [9], and which we follow here. More specifically, we endeavor to obtain a relationship between $V$ and $\dot{V}$ of the simple form $\dot{V} \leq-k V$. 
In the ordinary differential equation case, our viewpoint leads to an analysis of the matrix equation $A^{T} J+J A=-E$. It is well known that if $A$ is asymptotically stable, for any symmetric $E$ this equation has a unique symmetric solution $J$ and that the mapping $E \rightarrow J$ maps positive definite symmetric matrices into (but not onto) positive definite symmetric matrices. The following analysis is a generalization of this well-known result to the problem at hand.

LEMMA 2.2 The matrix problem

$$
\begin{gathered}
Q^{\prime}(\alpha)=C Q(\alpha)+\int_{0}^{\infty} D(\beta) Q(\alpha-\beta) d \beta, \quad \alpha>0, \quad Q(\alpha)=Q^{T}(-\alpha), \quad \alpha \in \mathbb{R}, \\
C Q(0)+Q(0) C^{T}+\int_{0}^{\infty}\left[D(\beta) Q^{T}(\beta)+Q(\beta) D^{T}(\beta)\right] d \beta=X,
\end{gathered}
$$

where $C$ is a constant matrix, $X$ a symmetric constant matrix and $D(\cdot) e^{\gamma \cdot} \in L_{1}(0, \infty) \cap$ $L_{2}(0, \infty)$ for some $\gamma>0$, has no more than one exponentially decaying solution.

Proof. It suffices to prove that if $X=0(2.7)$ has no nontrivial exponentially decaying solutions. Suppose that $Q$ is a solution with $|Q(\alpha)| \leq k e^{-v|\alpha|}$ for some $k, v>0$. Define $\hat{Q}(s)=\int_{-\infty}^{\infty} e^{-s \alpha} Q(\alpha) d \alpha$. Then $\hat{Q}$ is analytic in $s$ in the strip $|\operatorname{Re} s|<v$. Define $H(\alpha)$ for $\alpha \in \mathbb{R}$ by

$$
H(\alpha)=Q^{\prime}(\alpha)-C Q(\alpha)-\int_{0}^{\infty} D(\beta) Q(\alpha-\beta) d \beta
$$

it follows that $H(\alpha)=0$ for $\alpha \geq 0$ and that

$$
H(\alpha)=-C Q(\alpha)-Q(\alpha) C^{T}-\int_{0}^{\infty} D(\beta) Q(\alpha-\beta) d \beta-\int_{0}^{\infty} Q(\alpha+\beta) D^{T}(\beta) d \beta, \quad a \leq 0 .
$$

Extend the matrix function $D$ by $D(\beta)=0$ for $\beta<0$ and define $\hat{H}(s)$ and $\hat{D}(s)$ analogously to the definition of $\hat{Q}(s)$. It is easily seen that $\hat{H}$ is analytic and bounded in an open half-plane containing $\{s \mid \operatorname{Re} s \leq 0\}$ and that $\hat{D}$ is analytic and bounded in an open halfplane containing $\{s \mid \operatorname{Re} s \geq 0\}$. From (2.8) it follows that there is a strip about the imaginary axis in which

$$
\hat{H}(s)=\Delta(s) \hat{Q}(s), \quad \Delta(s)=s I-C-\hat{D}(s),
$$

and since $\hat{Q}(s)=\hat{Q}^{T}(-s)$ it follows that $\hat{H}(s) \Delta^{T}(-s)=\Delta(s) \hat{H}^{T}(-s)$ in this strip. Consider the function $F(s)=\hat{H}(s) \Delta^{T}(-s)$, which is analytic in an open half-plane containing $\{s \mid \operatorname{Re} s \leq 0\}$; the relation $F(s)=F^{T}(-s)$ shows that $F$ is an entire function. Moreover, it is easily seen that there exist positive constants $c_{1}$ and $c_{2}$ such that $|F(s)| \leq c_{1}+c_{2}|s|$ for all $s \in \mathbb{C}$. By a familiar extension of Liouville's theorem it follows that $F(s)=$ $F_{1}+s F_{2}$ for some complex matrices $F_{1}$ and $F_{2}$. Hence, $\hat{H}(s)=\left(F_{1}+s F_{2}\right)\left(-s I-C^{T}-\right.$ $\left.\hat{D}^{T}(-s)\right)^{-1}$ for $\operatorname{Re} s \leq 0$. From (2.7) and (2.9) it follows that $H^{\prime}(\alpha)$ exists and decays exponentially. Since $\lim _{\operatorname{Re} s \rightarrow-\infty} s \hat{H}(s)=H(0)$ and since $X=0$ implies $H(0)=0$, it follows that $F_{1}=F_{2}=0$. Thus $\hat{H}(s)$ vanishes identically, which implies that $\hat{Q}(s)$ and $Q(\alpha)$ must be zero, proving the lemma.

Let us now return to the construction of the Liapunov functional for Eq. (2.1). For this purpose, assume that for the given $A, B$ and $\delta$ Eq. (2.2) is uniformly stable and that the kernel $B(\cdot) e^{\delta \cdot} \in L_{1}(0, \infty) \cap L_{2}(0, \infty)$. Let $\varepsilon>0$, and consider the problem, obviously 
related to equation (2.7), of finding a function $Q$ within the class of exponentially decaying matrices defined by

$$
\begin{gathered}
Q^{\prime}(\alpha)=\left[A^{T}+(\delta-\varepsilon) I\right] Q(\alpha)+\int_{0}^{\infty} e^{(\delta-\varepsilon) \beta} B^{T}(\beta) Q(\alpha-\beta) d \beta, \quad \alpha \geq 0, \\
Q(\alpha)=Q^{T}(-\alpha), \quad \alpha \in \mathbb{R}, \\
A^{T} Q(0)+Q(0) A+\int_{0}^{\infty} e^{(\delta-\varepsilon) \beta}\left[B^{T}(\beta) Q^{T}(\beta)+Q(\beta) B(\beta)\right] d \beta=W,
\end{gathered}
$$

for some fixed but arbitrary positive definite matrix $W$, to be selected in the sequel. Lemma 2.2 above guarantees that, among exponentially decaying matrices, there is at most one $Q$ satisfying this equation. With $S_{\delta-\varepsilon}(t)=e^{(\delta-\varepsilon) t} S(t)$, where $S$ is the solution of Eq. (2.3), it is easily checked that

$$
Q(\alpha)=\int_{0}^{\infty} S_{\delta-\varepsilon}^{T}(u+\alpha) W S_{\delta-\varepsilon}(u) d u, \quad \alpha \in \mathbb{R},
$$

yields a solution of (2.11), hence the unique exponentially decaying solution of this equation. It is this matrix function $Q$ that allows us to construct a simple Liapunov functional of the form (2.5). Indeed, in that equation, let the matrices $L, H(\theta), N(\theta)$ and $P(\theta, \eta)$ be defined by

$$
\begin{gathered}
L=Q(0)+M, \\
H(\theta)=\operatorname{Re}^{2(\delta-\varepsilon) \theta} \\
N(\theta)=\int_{\theta}^{\infty} e^{(\delta-\varepsilon)(v+\theta)} Q(v+\theta) B(v) d v, \\
P(\theta, \eta)=\int_{-\theta}^{\infty} \int_{-\eta}^{\infty} e^{(\delta-\varepsilon)(\eta+\theta+u+v)} B^{T}(v) Q(\eta-\theta+u-v) B(u) d u d v,
\end{gathered}
$$

where the positive semidefinite matrices $M$ and $R$ will be chosen later. A lengthy computation yields that, with these matrices, Eq. (2.6) reduces to

$$
\begin{aligned}
\dot{V}\left(t, x_{t}(0), x_{t}\right)= & -2(\delta-\varepsilon) V\left(t, x_{t}(0), x_{t}\right) \\
& +x_{t}^{T}(0)\left[-W+R+\left(A^{T}+(\delta-\varepsilon) I\right) M+M(A+(\delta-\varepsilon) I)\right] x_{t}(0) \\
& +2 x_{t}^{T}(0) \int_{-t}^{0} M B(-\theta) x_{t}(\theta) d \theta .
\end{aligned}
$$

Moreover, it is possible to expose the structure of the functional $V$ under these conditions. Substitution of Eq. (2.12) into Eqs. (2.13)-(2.16) shows, after some appropriate rearrangements, that the functional $V$ is of the form

$$
\begin{aligned}
V\left(t, x_{t}(0), x_{t}\right)= & x_{t}^{T}(0) M x_{t}(0)+\int_{-t}^{0} x_{t}^{T}(\theta) R x_{t}(\theta) e^{2(\delta-\varepsilon) \theta} d \theta \\
+ & \int_{0}^{\infty}\left(S(\rho) x_{t}(0)+\int_{-t}^{0} \int_{-\theta}^{\infty} S(\rho-v-\theta) B(v) x_{t}(\theta) d v d \theta\right)^{T} W e^{2(\delta-\varepsilon) \rho} \\
& \times\left(S(\rho) x_{t}(0)+\int_{-t}^{0} \int_{-\theta}^{\infty} S(\rho-v-\theta) B(v) x_{t}(\theta) d v d \theta\right) d \rho,
\end{aligned}
$$


which can be rewritten, on account of Lemma 2.1, as

$$
\begin{aligned}
& V\left(t, x_{t}(0), x_{t}\right)=x_{t}^{T}(0) M x_{t}(0)+\int_{-t}^{0} x_{t}^{T}(\theta) R x_{t}(\theta) e^{2(\delta-\varepsilon) \theta} d \theta \\
&+\int_{0}^{\infty} x_{t}^{T}(\rho) W x_{t}(\rho) e^{2(\delta-\varepsilon) \rho} d \rho .
\end{aligned}
$$

Three points seem worthy of some emphasis at this point. The first is that whereas Eq. (2.5) is a very general form, it is the particular restriction of this form given by (2.12)-(2.16) that yields the simple, manageable results expressed by Eqs. (2.17) and (2.19). The second remark is that the simplest computable result for (2.17) is obtained for the case $\delta-\varepsilon=0$, $M=R=0$, in which case the sign of $\dot{V}$ is strictly given by $W$; but in this case, all that one can say is that $V$ is positive and difficult to estimate. Finally, if in (2.19) one chooses $W=0$, then one recovers the commonly used functionals for this problem, but then note that (2.16) is difficult if not impossible to estimate appropriately unless the matrix $A$ is asymptotically stable.

The emphasis here is to obtain estimates through appropriate use of the matrices $W$, $M$ and $R$. First note that with $W>0$, it follows from (2.19) that

$$
V\left(t, x_{t}(0), x_{t}\right) \geq x_{t}^{T}(0) M x_{t}(0)+\int_{-t}^{0} x_{t}^{T}(\theta) R x_{t}(\theta) e^{2(\delta-\varepsilon) \theta} d \theta .
$$

Let now $|\xi|_{M}=\left(\xi^{T} M \xi\right)^{1 / 2}$ for $\xi \in \mathbb{R}^{n}$, with the same notation for the corresponding matrix norm. Now, let $\varepsilon>0$ be given. Observe that

$$
\begin{aligned}
\left|2 x_{t}^{T}(0) \int_{-t}^{0} M B(-\theta) x_{t}(\theta) d \theta\right| \leq & 2\left|x_{t}(0)\right|_{M}\left(\int_{-t}^{0}\left|x_{t}(\theta)\right|_{M}^{2} e^{2(\delta-\varepsilon) \theta} d \theta\right)^{1 / 2} \\
& \times\left(\int_{-t}^{0}|B(-\theta)|_{M}^{2} e^{-2(\delta-\varepsilon) \theta} d \theta\right)^{1 / 2} \\
\leq \| & |B(\cdot)|_{M} e^{(\delta-\varepsilon) \cdot} \|_{L_{2}(0, \infty)}\left(c\left|x_{t}(0)\right|_{M}^{2}+\frac{1}{c} \int_{-t}^{0}\left|x_{t}(\theta)\right|_{M}^{2} e^{2(\delta-\varepsilon) \theta} d \theta\right)
\end{aligned}
$$

for every $c>0$. Hence, if we choose $c=\varepsilon /\left\|\mid B(\cdot) e^{(\delta-\varepsilon) \cdot}\right\|_{L_{2}}$ and $R=\left[\left(\left\|B(\cdot) e^{(\delta-\varepsilon) \cdot}\right\|_{L_{2}}^{2}\right)\right.$ $\left./ \varepsilon^{2}\right] M$, it follows from the form of $(2.19)$ that

$$
2\left|x_{t}^{T}(\theta) \int_{-t}^{0} M B(-\theta) x_{t}(\theta) d \theta\right| \leq 2 \varepsilon V\left(t, x_{t}(0), x_{t}\right) .
$$

Thus with this choice of $R$, and $M$ an arbitrary positive definite matrix, (2.17) yields

$$
\begin{aligned}
\dot{V}\left(t, x_{t}(0), x_{t}\right) \leq- & 2(\delta-2 \varepsilon) V\left(t, x_{t}(0), x_{t}\right) \\
+ & x_{t}^{T}(0)\left[-W+R+\left(A^{T}+(\delta-\varepsilon) I\right) M+M(A+(\delta-\varepsilon) I)\right] x_{t}(0)
\end{aligned}
$$

so that $W$ can be chosen as a positive definite matrix to yield

$$
\dot{V}\left(t, x_{t}(0), x_{t}\right) \leq-2(\delta-2 \varepsilon) V\left(t, x_{t}(0), x_{t}\right) .
$$


We may appeal to the definitions of $Q, N$, and $P$ to show that for some $k>0$ independent of $t$,

$$
V\left(t, x_{t}(0), x_{t}\right) \leq k\left(x_{t}^{T}(0) M x_{t}(0)+\int_{-t}^{0} x_{t}^{T}(\theta) R x_{t}(\theta) e^{2(\delta-\varepsilon) \theta} d \theta\right)
$$

Along with (2.20), this establishes just in what sense $V$ measures the size of the solution. For each fixed $t, V^{1 / 2}(t, \cdot, \cdot)$ is equivalent to the norm $\|\cdot\|_{t}$ given by

$$
\left\|\left(x_{t}(0), x_{t}\right)\right\|_{t}^{2}=\left|x_{t}(0)\right|^{2}+\int_{-t}^{0}\left|x_{t}(\theta)\right|^{2} e^{2(\delta-\varepsilon) \theta} d \theta .
$$

It can be said that $V^{1 / 2}(t, \cdot, \cdot)$ is equivalent to the usual norm on $\mathbb{R}^{n} \times L_{2}(-t, 0)$; however, the constants describing this relationship behave badly as $t$ grows, whereas the constants relating $V^{1 / 2}(t, \cdot, \cdot)$ to $(2.23)$ are independent of $t$. The preceding results are summarized in the following

TheOREM 2.1. Consider the equation $\dot{x}(t)=A x(t)+\int_{0}^{t} B(t-\tau) x(\tau) d \tau$ and the functional $V$ given by (2.5). Suppose that $\delta \in \mathbb{R}$ is such that $B\left(1^{\cdot}\right) e^{\delta \cdot} \in L_{1}(0, \infty) \cap L_{2}(0, \infty)$ and the equation $\dot{y}(t)=(A+\delta I) y(t)+\int_{0}^{t} e^{\delta(t-\tau)} B(t-\tau) y(\tau) d \tau$ is uniformly stable. Then for any $\varepsilon>0$ there exist continuous matrices $N, P$, and $H$, with $H$ positive definite, a positive definite matrix $L$, and $k_{2} \geq k_{1}>0$ such that

$$
\begin{aligned}
k_{1}\left(\left|x_{t}(0)\right|^{2}+\int_{-t}^{0}\left|x_{t}(\theta)\right|^{2} e^{2(\delta-\varepsilon) \theta} d \theta\right)^{1 / 2} & \leq V^{1 / 2}\left(t, x_{t}(0), x_{t}\right) \\
& \leq k_{2}\left(\left|x_{t}(0)\right|^{2}+\int_{-t}^{0}\left|x_{t}(\theta)\right|^{2} e^{2(\delta-\varepsilon) \theta} d \theta\right)^{1 / 2}
\end{aligned}
$$

and $\dot{V} \leq-2(\delta-2 \varepsilon) V$.

If $\delta>0$, the problem is exponentially asymptotically stable and $V$ yields the estimate $\left|x_{t}(0)\right| \leq c_{0} e^{-(\delta-2 \varepsilon)\left(t-t_{0}\right)}$ for a constant $c_{0}$ depending on the initial data on $\left[0, t_{0}\right]$ giving rise to $x$.

Remarks. The construction of a Liapunov functional for the equation

$$
\dot{x}(t)=A x(t)+\int_{t-r}^{t} B(t-\tau) x(\tau) d \tau,
$$

where $0<r<\infty$ and $B \in L_{2}(0, r)$, is carried out in exactly the same manner used above. The result is, in fact, more satisfactory. We regard (2.25) as an autonomous evolution in the Hilbert space $\mathbb{R} \times L_{2}(-r, 0)$. If the spectrum of the generator of the appropriate semigroup lies in the closed half-plane $\operatorname{Re} s \leq \delta_{0}$, then for any $\varepsilon_{0}>0$ we can construct a quadratic form $V_{0}$ on $\mathbb{R}^{n} \times L_{2}(-r, 0)$ such that $V_{0}^{1 / 2}$ is equivalent to the usual norm and $\dot{V}_{0} \leq 2\left(\delta_{0}+\varepsilon_{0}\right) V_{0}$.

The approach taken here may be of more general use. The linearity and appearance of the equation under consideration suggest a quadratic Liapunov functional. Above, the attempt to obtain a certain appearance in its derivative required that terms in (2.5) and (2.6) be comparable. In general, a set of equations will arise from the comparison of $\dot{V}$ to $V$, and various tactics are available. For example, dropping the requirement $P(\theta, \eta)=$ 
$P^{T}(\eta, \theta)$ leads to a functional which is useful for an equation whose kernel is not of convolution type. This remark is pursued in the following section.

3. More general equations. We now consider the equation in $\mathbb{R}^{n}$

$$
\dot{x}(t)=A(t) x(t)+\int_{0}^{t} K(t, \tau) x(\tau) d \tau,
$$

where $A$ and $K$ are continuous $n \times n$ matrices. The initial-value problem to be considered has $t_{0} \geq 0$ and $x=f$ on $\left[0, t_{0}\right], f \in C\left[0, t_{0}\right]$, with (3.1) to hold in $t \geq t_{0}$. Again, for $t \geq t_{0}$ we think of $\left(x_{t}(0), x_{t}\right)$ as lying in $\mathbb{R}^{n} \times L_{2}(-t, 0)$, and seek a quadratic Liapunov functional. Burton $[6,7]$ and others $[3,4,5]$ have studied $(3.1)$ by picturing it as a perturbed version of an ordinary differential equation, and we do so as well. The theorem presented in this section is said to be a Razumikhin-type result because its proof relies only on the description of certain solutions at the times those solutions leave sets which are of interest.

Before presenting the theorem, we recall a simple fact from linear algebra which can be found, for example, in [14]. If $D$ and $E$ are real symmetric $n \times n$ matrices with $E$ positive definite, the equation $\operatorname{det}[D-\lambda E]=0$ has $n$ real solutions $\lambda_{i}$. They coincide with the eigenvalues of $D E^{-1}$ and

$$
\max _{1 \leqslant i \leqslant n} \lambda_{i}=\max _{z \neq 0} \frac{z^{T} D z}{z^{T} E z} .
$$

As before, for $E>0$ and $\xi \in \mathbb{R}^{n}$, we write $|\xi|_{E}=\left(\xi^{T} E \xi\right)^{1 / 2}$, with the same notation for the corresponding matrix norm. If $G$ is an $n \times n$ matrix with real eigenvalues, let $\lambda_{\max }(G)$ denote its largest eigenvalue.

THEOREM 3.1. Suppose $M$ is a positive definite $n \times n$ matrix and let $g(t)=\lambda_{\max }\left(A^{T}(t)+\right.$ $\left.M A(t) M^{-1}\right)$. Suppose that there exists $\alpha>0$ such that

$$
2 \int_{0}^{t} \int_{0}^{u}|K(u, s)|_{M}\left(\exp \int_{u}^{t} g\right) d s d u \leq 1-\alpha
$$

for all $t \geq 0$. Then

(i) (3.1) is uniformly stable if there exists $N<+\infty$ such that $\int_{t}^{T} g(\tau) d \tau \leq N$ whenever $0 \leq t \leq T<+\infty$;

(ii) (3.1) is stable if lim $\sup _{T \rightarrow \infty} \int_{0}^{T} g(\tau) d \tau<+\infty$.

Proof. Let

$$
V_{1}(t, x(t), x)=x^{T}(t) M x(t)-2 \int_{0}^{t} \int_{0}^{u} x^{T}(u) M K(u, s) \exp \left(\int_{u}^{t} g\right) x(s) d s d u .
$$

Then along solutions of (3.1),

$$
\dot{V}_{1}(t, x(t), x)=x^{T}(t)\left[A^{T}(t) M+M A(t)-g(t) M\right] x(t)+g(t) V_{1}(t, x(t), x) .
$$

Thus $\dot{V}_{1}(t, x(t), x) \leq g(t) V_{1}(t, x(t), x)$. So, for $t \geq t_{0}$,

$$
V_{1}(t, x(t), x) \leq V_{1}\left(t_{0}, f\left(t_{0}\right), f\right) \exp \int_{t_{0}}^{t} g(\tau) d \tau,
$$


where $f$ is the initial data for $x$. If (3.1) were not uniformly stable, we could find sequences as follows: $\left\{t_{k}\right\}_{k=1}^{\infty}, t_{k} \geq 0,\left\{f_{k}\right\}_{k=1}^{\infty}, f_{k} \in C\left[0, t_{k}\right], \sup _{\left[0, t_{k}\right]}\left|f_{k}(t)\right|_{M} \leq 1$, and $\left\{T_{k}\right\}_{k=1}^{\infty}, T_{k} \geq t_{k}$, such that $\left|x_{k}\left(T_{k}\right)\right|_{M} \geq k$ and $\left|x_{k}(t)\right|_{M}<k$ for $t<T_{k}$, where $x_{k}$ is the solution of (2.7) arising from initial data $f_{k}$ on $\left[0, t_{k}\right]$. For any $k \geq 1$,

$$
\begin{aligned}
V_{1}\left(T_{k}, x_{k}\left(T_{k}\right), x_{k}\right) & \geq\left|x_{k}\left(T_{k}\right)\right|_{M}^{2}-2 \int_{0}^{T_{k}} \int_{0}^{u}\left|x_{k}(u)\right|_{M}|K(u, s)|_{M} \exp \left(\int_{u}^{T_{k}} g\right)\left|x_{k}(s)\right|_{M} d s d u \\
& \geq\left|x_{k}\left(T_{k}\right)\right|_{M}^{2}\left(1-2 \int_{0}^{T_{k}} \int_{0}^{u}|K(u, s)|_{M} \exp \left(\int_{u}^{T_{k}} g\right) d s d u\right) \\
& \geq \alpha k^{2} .
\end{aligned}
$$

Also,

$$
\begin{aligned}
V_{1}\left(t_{k}, f_{k}\left(t_{k}\right), f_{k}\right) & \leq\left|f_{k}\left(t_{k}\right)\right|_{M}^{2}+\left.2 \int_{0}^{t_{k}} \int_{0}^{u}\left|f_{k}(u)\right|_{M}|| K(u, s)\right|_{M} \exp \left(\int_{u}^{t_{k}} g\right)\left|f_{k}(s)\right|_{M} d s d u \\
& \leq 1+2 \int_{0}^{t_{k}} \int_{0}^{u}|K(u, s)|_{M} \exp \left(\int_{u}^{t_{k}} g\right) d s d u \\
& \leq 2-\alpha .
\end{aligned}
$$

From (3.5) applied to these inequalities, we obtain

$$
\alpha k^{2} \leq(2-\alpha) \exp \int_{t_{k}}^{T_{k}} g(\tau) d \tau .
$$

Since (3.6) must hold for every $k, \lim _{k \rightarrow \infty} \int_{t_{k}}^{T_{k}} g(\tau) d \tau=+\infty$. This proves (i).

To prove (ii) we follow the same argument except that all the $t_{k}$ coincide at some $t_{0} \geq 0$. The same chains of inequalities lead us to this version of (3.6):

$$
\alpha k^{2} \leq(2-\alpha) \exp \int_{t_{0}}^{T_{k}} g(t) d t .
$$

This implies that lim $\sup _{t \rightarrow \infty} \int_{0}^{t} g(\tau) d \tau=+\infty$ and thus establishes (ii).

Remarks. The formulas in (3.3) and (3.4) resemble those describing $V$ and $\dot{V}$ in Sec. 2. In fact, $V_{1}$ is motivated by the construction there. If $K(t, \tau)=B_{1}(t-\tau)$ and $A(t) \equiv A_{1}$, then $g(t) \equiv g_{1}$ and the final term in (3.3) takes the form

$$
-2 \int_{-t}^{0} \int_{-t}^{0} x_{t}^{T}(\theta) M B_{1}(\theta-\eta) e^{-g_{1} \theta} x_{t}(\eta) d \eta d \theta
$$

after a change of variables. It may be rewritten as

$$
\int_{-t}^{0} \int_{-t}^{0} x_{t}^{T}(\theta) P_{1}(\theta, \eta) x_{t}(\eta) d \eta d \theta
$$

with $P_{1}(\theta, \eta)=-M B_{1}(\theta-\eta) e^{-g_{1} \theta}$ for $-t \leq \eta<\theta \leq 0$ and $P_{1}(\eta, \theta)=P_{1}^{T}(\theta, \eta)$. Then

$$
\frac{\partial P_{1}(\theta, \eta)}{\partial \theta}+\frac{\partial P_{1}(\theta, \eta)}{\partial \eta}=-g_{1} P_{1}(\theta, \eta)
$$

so that $V_{1}$ fits the term-by-term comparison scheme used before; the kernel corresponding to $N$ of Sec. 2 vanishes. 
The simple appearance of $V_{1}$ and $\dot{V}_{1}$ does not even depend on the linearity of (3.1). However, we have not as yet thoroughly exploited this. Functionals similar to $V_{1}$ appear in Miller [12] for the case of a scalar equation.

The functional $V_{1}$ may be useful in analyzing equations whose kernels do not decay exponentially, provided the equation can be viewed as a perturbation of a suitably stable ordinary differential equation. The following example illustrates this. Consider (3.1) where $\left.n=2,|K(t, \tau)| \leq k_{0} \min (1, t-\tau)^{-2}\right)$, and

$$
A(t)=\left[\begin{array}{rr}
-1 & \beta(t) \\
0 & -1
\end{array}\right]
$$

where $|\beta(t)| \leq 2-\beta_{0}$ for some fixed $\beta_{0}>0$. We apply Theorem 3.1 with $M=I$. Then $g(t)=-2+|\beta(t)| \leq-\beta_{0}$. Moreover,

$$
\begin{aligned}
& 2 \int_{0}^{t} \int_{0}^{u}|K(u, s)| \exp \left(\int_{u}^{t}-2+|\beta(\tau)| d \tau\right) d s d u \\
& \quad \leq 2 \int_{0}^{t} e^{-\beta_{0}(t-u)}\left(\int_{0}^{u}|K(u, s)| d s\right) d u<4 k_{0} \int_{0}^{t} e^{-\beta_{0}(t-u)} d u \leq 4 k_{0} \beta_{0}^{-1},
\end{aligned}
$$

and the equation is uniformly stable if $4 k_{0} \leq \beta_{0}$.

\section{REFERENCES}

[1] R. K. Miller, Asymptotic stability properties of linear Volterra integrodifferential equations, J. Diff. Eq. 10, 485-506 (1971)

[2] S. I. Grossman and R. K. Miller, Nonlinear Volterra integrodifferential systems with L'-kernels, J. Diff. Eq. 13, 551-566 (1973)

[3] G. Seifert, Liapunov-Razumikhin conditions for stability and boundedness of functional differential equations of Volterra type, J. Diff. Eq. 14, 424-430 (1973)

[4] G. Seifert, Liapunov-Razumikhin conditions for asymptotic stability in functional differential equations of Volterra type, J. Diff. Eq. 16, 289-297 (1974)

[5] R. Grimmer and G. Seifert, Stability properties of Volterra integrodifferential equations, J. Diff. Eq. 19, 142-166 (1973)

[6] T. A. Burton, Stability theory for Volterra equations, J. Diff. Eq. 32, 101-118 (1979)

[7] T. A. Burton, Uniform stabilities for Volterra equations, J. Diff. Eq. 36, 40-53 (1980)

[8] E. F. Infante and W. B. Castelan, A Liapunov functional for a matrix neutral difference-differential equation with one delay, J. Math. Anal. Appl. 71, 105-130 (1979)

[9] E. F. Infante and W. B. Castelan, A Liapunov functional for a matrix difference-differential equation, J. Diff. Eq. 29, 439-451 (1978)

[10] E. F. Infante and W. B. Castelan, On a functional equation arising in the stability theory of differencedifferential equations, Quart. Appl. Math. 35, 311-319 (1977)

[11] L. A. V. Carvalho, E. F. Infante, and J. A. Walker, On the existence of simple Liapunov functions for linear retarded difference-differential equations, Tôhoku Math. J. 32, 283-297 (1980)

[12] R. K. Miller, Nonlinear Volterra integral equations, W. A. Benjamin, Inc., Menlo Park, CA, 1971

[13] J. K. Hale, Theory of functional differential equations, Springer-Verlag, New York, 1977

[14] F. R. Gantmacher, The theory of matrices, Vol. 1, Chelsea, New York, 1977 